\title{
Ocular signs and symptoms and vitamin A status in patients with cystic fibrosis treated with daily vitamin A supplements
}

Ejaz A Ansari, Kamal Sahni, Christine Etherington, Alison Morton, Steven P Conway, Eduardo Moya, James M Littlewood

\begin{abstract}
Backgroundlaims-Patients with cystic fibrosis (CF) may have low plasma vitamin A levels from malabsorption, zinc deficiency, liver disease, or poor compliance with prescribed supplements. In view of the increasing number of adults with $\mathrm{CF}$, many of whom drive cars, it is important to assess vitamin A status. In our centre an attempt has been made to achieve normal levels of fat soluble vitamins by annual estimation of plasma levels and appropriate oral supplementation. This study aimed to determine if this approach prevents vitamin A deficiency and the consequent problems with dark adaptation.
\end{abstract}

Methods-The study was conducted at the regional adult and paediatric cystic fibrosis unit and the patients were recruited from there. Dark adaptation studies were conducted at the department of ophthalmology, St James's University Hospital. All patients are regularly seen in the outpatient department by a CF specialist dietitian and have a comprehensive annual dietary assessment. 28 patients had the following investigations: serum retinol, plasma zinc, serum retinol binding protein, liver function tests, dark adaptation, contrast sensitivity, and anterior ocular surface status. 25 age and sex matched controls without CF or ocular pathology were also recruited for the dark adaptation study.

Results-None of the patients had vitamin A deficiency, the median value of serum retinol being $48 \mu \mathrm{g} / \mathrm{dl}$, range $31-80 \mu \mathrm{g} / \mathrm{dl}$ (normal range 30-80 $\mu$ g/dl). Dark adaptation was normal in all cases compared with the control group where the mean value was $3.4 \mathrm{log}$ units of threshold luminance $(95 \%$ confidence interval $2.4-$ 4.0). None of the test group had a value of threshold luminance $2 \mathrm{SD}$ above the mean value for the control group. Eight patients had reduced contrast sensitivity. The median value for serum zinc was 14.2 $\mu \mathrm{mol} / 1$, range $13-81 \mu \mathrm{mol} / 1$ (normal range 8-23 $\mu \mathrm{mol} / \mathrm{l})$ and the median value for retinol binding protein was $36 \mathrm{mg} / 1$, range 13-81 $\mathrm{mg} / 1$ (normal range $35-58 \mathrm{mg} / \mathrm{l}$ ). There was no correlation between dark adaptation and serum retinol, zinc, or retinol binding protein. Two patients had clinical evidence of dry eye.
Conclusion-Regular estimates of plasma vitamin A together with appropriate supplementation and expert dietetic review can maintain normal dark adaptation in patients with cystic fibrosis. The occurrence of reduced contrast sensitivity function is well documented but remains an unexplained phenomenon and deserves further study.

(Br F Ophthalmol 1999;83:688-691)

It has been reported that a proportion of cystic fibrosis (CF) patients have abnormal dark adaptation and conjunctival xerosis as a result of vitamin A deficiency. ${ }^{1}$ The affected patients had lower plasma vitamin A levels and were more likely to have abnormal liver function tests than CF patients with normal vitamin A levels. These patients were considered to have received an adequate vitamin $\mathrm{A}$ intake with a daily supplement of 5000 IU, although the duration of this supplementation was not stated and compliance may have been poor.

Another report in which the patients received similar vitamin A supplementation to those in the above study showed that none of the patients had clinical or biological signs of vitamin A deficiency. A higher vitamin A intake from the diet and better pancreatic enzyme replacement were suggested as being responsible for the better vitamin A status. ${ }^{2}$

Some patients with abnormal dark adaptation do not respond to increasing their vitamin A intake until zinc supplements are administered. A relation between vitamin A and zinc is well documented. ${ }^{34}$ Low zinc levels are unusual in our experience. ${ }^{5}$

The nutritional care $\mathrm{CF}$ patients receive at the Leeds regional paediatric and adult $\mathrm{CF}$ unit may well be different from other centres as we have been aware of the need for regular supplementation of fat soluble vitamins for the past 15 years. ${ }^{6}$ We might hope that with the regular specialised dietary advice which the Leeds CF patients have received and the regular monitoring of their intestinal malabsorption and plasma vitamin A levels, they may be in a better nutritional state than patients described in previous studies. ${ }^{78}$

Vitamin A deficiency appears to be potentially common in CF patients and the topic has been reviewed by Eid and associates. ${ }^{9}$ In view of the improved survival and increasing number of $\mathrm{CF}$ adults, many of whom drive cars, it is important to assess the vitamin A status of patients attending a CF centre where an
Accepted for publication 10 December 1998 
attempt has been made to achieve normal levels of fat soluble vitamins by periodic estimation of plasma levels and appropriate oral supplementation. We need to determine if our approach, described above, prevents vitamin A deficiency and the consequent problems with dark adaptation.

\section{Patients and methods}

Twenty eight patients (20 males and eight females) from the regional adult and paediatric cystic fibrosis unit in Leeds were recruited for this study which had ethics committee approval. The patients or their guardians had been informed about the study and consent was obtained from all participants. The age range was $12-33$ years, median 20 years. The $\mathrm{CF}$ diagnosis was made on all the patients with two positive sweat tests and/or identifiable CF mutations. All patients are supplemented from diagnosis, usually from under 1 year of age. At our centre all patients are regularly monitored by a CF specialist and dietitian at least every 2 months and have a comprehensive assessment every $12-18$ months. This includes a prospective dietary diary analysis. All patients initially receive $8000 \mathrm{IU}$ of vitamin A daily. All patients were taking pancreatic enzyme supplements. Fasting blood is sampled for vitamin A measurement at assessment and the vitamin $\mathrm{A}$ dose adjusted if necessary. At other times patients with any dietary or malabsorption problems have ready access to the specialist dietitian. Malabsorption is screened for at times other than the annual assessment when clinically indicated by stool fat microscopy, faecal pancreatic elastase, and faecal chymotrypsin levels. The control group consisted of 25 healthy age and sex matched individuals without a family or personal history of $\mathrm{CF}$, malabsorption, other gastrointestinal/hepatic disease or eye disorders.

Venous blood was taken in the morning after overnight fasting for serum vitamin A estimation. The blood was shielded from light and high performance liquid chromatography was used to measure serum retinol concentration. Venous blood was also taken for measurement of serum zinc, retinol binding protein (RBP), and liver function tests (LFTs).

Dark adaptation was tested using the Friedmann Field Analyser Mk 1 with an auxiliary filter bar. ${ }^{10}$ The pupils were fully dilated for the study with tropicamide $1 \%$ and phenylephrine $2.5 \%$.

Table 1 Values for the variables tested

\begin{tabular}{llll}
\hline & Median & Range & Normal range \\
\hline Age (years) & 20 & $12-33$ & \\
Weight $(\mathrm{kg})$ & 57 & $34.8-69$ & \\
BMI & 19.3 & $15.3-23.8$ & $18.5-25$ \\
Serum vitamin A $(\mu \mathrm{g} / \mathrm{dl})$ & 48 & $31-80$ & $30-80$ \\
Serum zinc $(\mu \mathrm{mol} / \mathrm{l})$ & 14.2 & $7.7-58.6$ & $8-23$ \\
Retinol binding protein $(\mathrm{mg} / \mathrm{l})$ & 36 & $13-81$ & $35-56$ \\
Bilirubin $(\mu \mathrm{mol} / \mathrm{l})$ & 11 & $3-26$ & $3-15$ \\
Apartate aminotransferase $(\mathrm{IU} / \mathrm{l})$ & 22 & $15-81$ & $0-50$ \\
Alanine aminotransferase $(\mathrm{IU} / \mathrm{l})$ & 12 & $10-91$ & $0-35$ \\
Globulin $(\mathrm{g} / \mathrm{l})$ & 39 & $30-50$ & $16-37$ \\
Alkaline phosphatase $(\mathrm{IU} / \mathrm{l})$ & 223 & $69-867$ & $90-230$ \\
Albumin $(\mathrm{g} / \mathrm{l})$ & 34 & $25-38$ & $30-55$ \\
Total protein $(\mathrm{g} / \mathrm{l})$ & 73 & $63-85$ & $60-80$ \\
\hline
\end{tabular}

^ Body mass index.
Each eye was tested separately and was initially light adapted by setting off the flash of an ordinary photographic flash lamp. All external light sources were put off so that the examination room was in total darkness. Every minute the threshold sensitivity of the eye was measured by firing a light stimulus and adjusting the filter density until the stimulus was just perceived. This was plotted on the dark adaptation curve. This was repeated for 30 minutes and the dark adaptation curve completed. Testing of the fellow eye was then commenced.

Contrast sensitivity was assessed using Vistech charts in optimal daylight conditions and with refractive correction if appropriate. Five spatial frequencies were tested ranging from 1.5-18 cycles per degree. The external surface of the eye was examined using the slit lamp. Tear break up time (TBUT) was measured to assess tear film stability. A value of $<10$ seconds was abnormal. Schirmer 1 test was used to estimate tear film quantity, a value of $<10 \mathrm{~mm}$ after 5 minutes was abnormal. Rose bengal $1 \%$ instilled into the inferior fornix was used to assess the amount of dead epithelial cells at the ocular surface. If two of these variables were abnormal then dry eye was the diagnosis. ${ }^{11}$

\section{STATISTICS}

Abnormal dark adaptation was present if the final threshold to the light target was $2 \mathrm{SD}$ above the mean of a control group of 25 patients. This group included 15 males and 10 females aged between 17 and 36 years with a median age of 22 years. The Pearson correlation coefficient was employed to study the correlation between dark adaptation, vitamin A, serum zinc, and retinol binding protein.

\section{Results}

Median vitamin A dose was 12000 units per day, range 8000-48 000. Median pancreatic lipase was $7500 \mathrm{U}$ lipase $/ \mathrm{kg} /$ day, range $8.75-$ 20 500. All patients were pancreatic insufficient. None of the patients had vitamin A deficiency, the median value being $48 \mu \mathrm{g} / \mathrm{dl}$, range 31-80 (normal range 30-80 $\mu \mathrm{g} / \mathrm{dl}$ ). Median serum zinc was $14.2 \mu \mathrm{mol} / 1$, range $7.7-58.6$ (normal range $8-23 \mu \mathrm{mol} / \mathrm{l}$ ). Only four patients had marginally low plasma zinc levels. Median $\mathrm{RBP}$ was $36 \mathrm{mg} / \mathrm{l}$, range 13-81 (normal range 35-58 mg/l). Only four patients had low RBP levels. Only two patients had abnormal LFTs. These results are summarised in Table 1 .

Stool fat microscopy in patients receiving pancreatic enzyme treatment showed excess fat in three patients. Only three patients complained of abdominal pain. No patient reported abnormal stool consistency. The body mass index (BMI) was used as a marker of nutritional status, the median value being 19.3, range 15.3-23.8 (normal range 18.5-25).

Dark adaptation was normal in all cases compared with the control group. The median value was $3.6 \mathrm{log}$ units of threshold luminance, range $2-4$. The control group had a median and mean value of $3.4 \mathrm{log}$ units of threshold luminance with a range of 2-4.2 (95\% 
Table 2 Correlation values between dark adaptation, serum vitamin $A$, serum zinc, and retinol binding protein using Pearson's correlation coefficient $(r)$

\begin{tabular}{lcccl}
\hline Pearson's $r$ & Vitamin A & Zinc & $R B P$ & Dark adaptation \\
\hline Vitamin A & 1 & -0.04 & 0.42 & -0.16 \\
Zinc & -0.04 & 1 & -0.25 & -0.04 \\
RBP & 0.42 & -0.25 & 1 & -0.04 \\
Dark adaptation & -0.16 & -0.04 & -0.04 & 1 \\
\hline
\end{tabular}

confidence interval 2.4-4.0). There was no correlation between dark adaptation and serum retinol, zinc, or RBP, although a weak relation between vitamin A and RBP $(r=0.42$; $\mathrm{p}=0.07$ ) was present (Table 2). Eight patients had reduced contrast sensitivity on the Vistech contrast sensitivity evaluation form. The reduction was at the intermediate and high spatial frequencies. Two patients had evidence of dry eye, both having reduced tear film stability and dying epithelial cells.

\section{Discussion}

There was no evidence of biochemical or clinical vitamin A deficiency in our patients. Serum retinol was within the normal range and dark adaptation was normal. There was no correlation between dark adaptation and serum vitamin $\mathrm{A}, \mathrm{RBP}$, or serum zinc. In a study from Nottingham, $19 \%$ of patients had abnormal dark adaptation and $10 \%$ had frank conjunctival xerosis. ${ }^{1}$ All patients had oral pancreatic enzyme replacements but only an average intake of 5000 IU of vitamin A daily. It has been suggested that $25000-50000$ IU may be required to maintain liver storage of vitamin $\mathrm{A}$ in patients with $\mathrm{CF} .{ }^{12}$

In a more recent study by Morkeberg et al, all 35 adult patients had normal dark adaptation and similar vitamin A supplementation to the Nottingham patients. The better vitamin A status was explained by a higher dietary intake and better pancreatic enzyme replacement. ${ }^{2}$ Neither of these studies included estimation of serum zinc or whether this was routinely monitored. This is important as some patients with abnormal dark adaptation do not respond to vitamin A supplements until zinc supplements are given. ${ }^{3}$ From our study and Morkeberg et al's study it is difficult to ascertain whether higher dietary intake, pancreatic enzyme replacement, or vitamin A supplementation (or a combination of these) accounts for normal retinol levels and dark adaptation. However, both studies suggest that regular monitoring of serum retinol would be beneficial. Our study, in addition, suggests that vitamin A supplementation may have to be a lot more than 5000 IU in order to maintain normal retinol levels and differs from Morkeberg's findings in this respect. Therefore, all patients must be assessed individually and the relevant clinical and laboratory variables must be analysed in each case before deciding on the amount of supplementation rather than prescribing a uniform dosage for all patients.

The Nottingham group prescribed vitamin $\mathrm{E}$ for their patients as this has been shown to increase the absorption of vitamin $\mathrm{A} .{ }^{13}$ However, this could not have affected vitamin A status significantly in this group because supplementation of vitamin A itself at $5000 \mathrm{IU}$ daily was insufficient. There were no individual values given of serum retinol levels in these patients. In one case report of a 16 year old male CF patient, retinal function measured electrophysiologically only returned to normal with daily vitamin A supplementation of 25000 IU and even then the serum retinol level was low normal at $40 \mu \mathrm{g} / \mathrm{dl} .{ }^{14}$ This again substantiates the belief that in CF patients vitamin A supplementation needs to be considerably higher in order to maintain liver stores. Therefore, regular serum estimations of retinol and appropriate administration of adequate vitamin A supplements is most pertinent.

The contrast sensitivity function (CSF) is the ability of the visual system to detect information presented to the eye in the form of spatial or temporal contrast ${ }^{15}$ and may be a more sensitive indicator of optic nerve disease. ${ }^{16}$ Eight of our patients, all with corrected visual acuities of $6 / 6$ or better, had reduced contrast sensitivity at the intermediate and high spatial frequencies. Spaide and associates reported a decreased CSF in all 32 of their patient cohort. ${ }^{17}$ This was believed to be due to the use of chloramphenicol, but the CSF was reduced even in those patients who had not taken that antibiotic. It was concluded that chloramphenicol is not the only cause for the reduced CSF and other factors such as iatrogenic, nutritional, or primary effects of the disease itself could result in compromising the optic nerve and causing a decrease in CSF. It has been suggested that reduced CSF could be caused by subclinical retinal abnormalities resulting from vitamin A deficiency. ${ }^{14}$ However, all of our patients had good vitamin A status so the cause of the low CSF is probably not retinal. A more thorough study of CSF is required in patients with normal vitamin A status before we can confidently answer the question of the reason for reduced CSF.

Spaide et al also found a preganglionic oculosympathetic paresis of pupillary function in 10 out of 23 patients tested and this corresponded to the disease severity, as measured by the Schwachman score. ${ }^{17}$ Although we did not specifically test pupillary function, all our patients dilated well after instillation of tropicamide 1\% and phenylephrine $2.5 \%$ for the dark adaptation study. This may be due to overall healthier status, but we would have to dilate our patients' pupils with 5\% cocaine as in Spaide's study and use the Schwachman scoring system for our patients before true comparisons could be made.

Two of our patients had clinical evidence of dry eye according to the Copenhagen criteria. ${ }^{11}$ All of our patients had normal vitamin A status therefore the dry eye finding is unlikely to be due to vitamin A deficiency. None of the controls had dry eye. Morkeberg et al had similar conclusions although they had a greater incidence of dry eye. ${ }^{2}$ They suggested that the primary defect in exocrine secretion with osmolar derangements in CF could involve one or several parts of the tear production system. Histological examination of the ocular surface structures in vitamin A deficiency show 
squamous metaplasia of the conjunctival and corneal epithelia with keratinisation and reduced number of goblet cells. ${ }^{18}$ Morkeberg's group also looked at conjunctival cytology and could find no histological evidence of vitamin A deficiency. Therefore, they speculated that dry eye could be a direct manifestation of cystic fibrosis. Our study did not include conjunctival impression cytology, therefore we cannot say if our patients had histological evidence of vitamin A deficiency.

Thus, the achievement of normal levels of fat soluble vitamins by annual plasma estimation and appropriate oral supplementation with regular medical and dietary review and comprehensive dietary assessment prevents vitamin A deficiency and consequent problems with dark adaptation. The finding of dry eye in the presence of normal retinol levels confirms previous views that this may be a primary manifestation of cystic fibrosis. The occurrence of reduced contrast sensitivity function in patients with normal vitamin A status remains an unexplained phenomenon and deserves further study.

1 Neugebauer MA, Vernon SA, Brimlow G, et al. Nyctalopia and conjunctival xerosis indicating vitamin A deficiency in cystic fibrosis. Eye 1989;3:360-4.

2 Morkeberg JC, Edmund C, Prause JU, et al. Ocular findings in cystic fibrosis patients receiving vitamin A supplementation. Graefes Arch Clin Exp Ophthalmol 1995;233:709-13.
3 Keeling PW, O'Day J, Ruse W, et al. Zinc deficiency and photoreceptor dysfunction in chronic liver disease. Clin $\mathrm{Sci}$ 1982;62:109-11.

4 Smith JC Jr. The vitamin A-zinc connection: a review. Ann NY Acad Sci 1980;355:62-75.

5 Kelleher J, Goode HF, Field HP, et al. Essential element nutritional status in cystic fibrosis. Human Nutrition: Appl Nutr 1986;40A:79-84.

6 Congdon PJ, Bruce G, Rothburn MM, et al. Vitamin status in treated patients with cystic fibrosis. Arch Dis Child 1981; 56:708-14.

7 Littlewood JM, Kelleher J, Rawson I, et al. Comprehensive assessment at a CF centre identifies suboptimal treatment and improves management, symptoms and condition. 10th International Cystic Fibrosis Congress, Sydney, 1988.

8 Littlewood JM. Value of comprehensive assessment and investigation in management of cystic fibrosis. 18th European Cystic Fibrosis Conference, Madrid, 1993.

9 Eid NS, Shoemaker LR, Samiec TD. Vitamin A in cystic fibrosis: case report and review of the literature. 7 Paediatr Gastroenterol Nutr 1990;10:265-9.

10 Friedmann Analyser Instruction Manual. London: Clement Clarke International Ltd.

11 Manthorpe R, Oxholm P, Prause JU, et al. The Copenhagen criteria for Sjogren's syndrome. Scand F Rheumatol (suppl) 1986;61:19-21.

12 Sommer A. Vitamin A deficiency today. Conjunctival xerosis in cystic fibrosis. $\mathcal{F} R$ Soc Med 1989;82:1-2.

13 Ekvall S, Mitchell A. The effect of supplemental vitamin E on vitamin A serum levels in cystic fibrosis. Int $\mathcal{F}$ Vitamin Nutr Res 1978;48:324-32.

14 Leguire LE, Pappa KS, Kachmer ML, et al. Loss of contrast sensitivity in cystic fibrosis. Am f Ophthalmol 1991;111: $427-9$.

15 Sjostrand J, Abrahamsson M. Contrast sensitivity and disability glare. Nordisk Med 1992;107:316-18.

16 Kupersmith MJ, Nelson JI, Seiple WH, et al. The 20/20 eye in multiple sclerosis. Neurology 1983;33:1015-20.

17 Spaide RF, Diamond G, D'Amico RA, et al. Ocular findings in cystic fibrosis. Am f Ophthalmol 1987;103:204-10.

18 Watson NJ, Hutchinson CH, Atta HR. Vitamin A deficiency and xerophthalmia in the United Kingdom. BMF 1995; 310:1050-1 